\title{
Comparison between two databases regarding the classification of Drug Interactions and their mechanism of action
}

\section{Abstract}

Introduction Many drug-related problems are caused by drug interactions (DIs) that result from the pharmacological influence of one drug on another when taken together. Dls may have beneficial and predictable effects, but may also cause undesirable results, such as treatment ineffectiveness or serious adverse effects. There are a number of available databases with information about Dls. Although the information they provide is not always consensual, it is a useful tool in analysing the occurrence of potential drug interactions (PDIs) and in preventing unwanted or even fatal consequences for patients.

Objectives To compare the convergence of two databases (Micromedex/Drugs.com) regarding the classification of Dls and their mechanism of action.

Methodology A cross-sectional, descriptive and exploratory observational study was carried out using prescriptions from users with diabetes, dyslipidaemia or both pathologies from pharmacies in two districts, Porto and Braga. Information from each sample group was further analysed using two databases, Micromedex/Drugs.com.

Results Of the 57 pairs whose interaction potential was analysed, $64.3 \%$ converged on the classifications in both databases. Micromedex assigns moderate potential interaction to 36 drug pairs, and of these, 28 get the same rating on Drugs.com. Regarding the major PDIs, the degree of agreement was $40 \%$. There are 4 drug pairs with potential for major interaction at Micromedex to which Drugs.com assigns moderate PDI. Minor PDIs were found on Drugs.com in 4 drug pairs, which Micromedex rated as major or moderate. For the remaining twelve divergent rating pairs, Drugs.com does not assign them potential for interaction while Micromedex gives them potential for major or moderate PDIs. Regarding 
the consequences of the occurrence of PDIs, there is agreement between Micromedex and Drugs.com, except for pair AAS/Loop Diuretics.

Conclusion Micromedex identifies a higher number of PDIs and assigns higher severity to a higher number. 